\title{
Coalgebraic tensor product and homology operations
}

\author{
TAKUJI KASHIWABARA
}

In this paper, we show that Hunton-Turner coalgebraic tensor product respects various actions of Hopf algebras of homology operations.

16W30, 18G99, 55N20, 55S05, 55S10; 55S12, 55S25, 55U99, 57T05, 57T25

\section{Introduction}

In their paper [4] Hunton and Turner set up the framework for homological algebra of coalgebraic rings and coalgebraic modules, which arise naturally in the study of ordinary and generalized homologies of infinite loop spaces. Their language has proved to be a useful tool both for describing (see eg Hunton and Turner [5] and Kashiwabara and Wilson [7]) and computing (see Kashiwabara [6]) homology of infinite loop spaces. Of course, such objects tend to get equipped with extra structures. For example the Steenrod algebra [11], the Dyer-Lashof algebra [3] or the algebra of cohomology operations for appropriate generalized cohomology theory (see Adams [1]) may act on these objects. Thus it is natural to ask if these actions can be extended to the algebraic constructions defined by Hunton and Turner, notably to the coalgebraic tensor product.

The purpose of this paper is to answer this question, namely we prove:

Theorem 1.1 Let $\Theta$ be a Hopf algebra over the base-ring $R, A_{*, *}$ a commutative coalgebraic ring over $R, M_{*, *}, N_{*, *}$ coalgebraic modules over $A_{*, *}$. If $\Theta$ acts on all of $A_{*, *}, M_{*, *}$ and $N_{*, *}$ in a way that is compatible with their structure maps, then it also acts on $M_{*, *} \bar{\otimes}^{A, *} N_{*, *}$.

More precise statements will be given in later sections.

The paper is organized as follows. In Section 2, we recall necessary results from [4]. In Section 3 we treat the case of $M \bar{\otimes}^{R[S]} R[T]$, where $T$ is an $S$-module and $M$ is an $R[S]$-coalgebraic module, which is somewhat simpler than the rest. In Section 4, we treat the case of $M \bar{\otimes}^{R[S]} N$ where both $M$ and $N$ are arbitrary $R[S]$-coalgebraic modules, and the proof becomes more complicated. In Section 5, we treat the general case. The proof doesn't get more complicated, but the definition requires more care 
than in Section 4. Throughout the paper, $R$ will denote a commutative ring with unit (which may be graded).

This work is partially supported by ANR project number BLAN08-2_338236, HGRT.

\section{Basics on coalgebraic algebra}

In this section we quote relevant results of [4] for the convenience of the reader. For more details, refer to [4]. We start by quoting some definitions.

Definition 2.1 [4] (1) A graded abelian coalgebraic group over $R$ is an abelian group object in the category of graded cocommutative, coassociative coalgebras with counit over $R$.

(2) A bigraded commutative coalgebraic ring over $R$ is a graded commutative ring object in the category of graded cocommutative, coassociative coalgebras with counit over $R$.

As usual, we note by $*$ the "additive" products and $\circ$ the "multiplicative" products on such objects.

Remark 2.2 Coalgebraic rings were studied long before the works of Hunton and Turner [4], eg by May [8], Milgram [9] and Ravenel and Wilson [10]. Unfortunately their denominations (Hopf-ring or bialgebra) would cause us problems of internal coherence or compatibility with names in other domains. Thus we adopt the denomination in [4].

Definition 2.3 [4] An $A_{*, *}$ coalgebraic module over $R$ is a graded abelian coalgebraic group $M_{*, *}$ over $R$ equipped with a coalgebra map

$$
\circ: A_{*, *} \otimes{ }^{R} M_{*, *} \rightarrow M_{*, *}
$$

satisfying the following five axioms for all $a, b \in A_{*, *}$ and $x, y \in M_{*, *}$.

(1) $(a \circ b) \circ x=a \circ(b \circ x)$

(2) $[1] \circ x=x$

(3) $[0] \circ x=\eta \epsilon(x)$

(4) $(a * b) \circ x=\sum(-1)^{\left|x^{\prime}\right||b|}\left(a \circ x^{\prime}\right) *\left(b \circ x^{\prime \prime}\right)$

(5) $a \circ(x * y)=\sum(-1)^{\left|a^{\prime \prime}\right||x|}\left(a^{\prime} \circ x\right) *\left(a^{\prime \prime} \circ y\right)$

Here $\epsilon$ is the counit of $M_{*, *}, \eta$ is the coaugmentation, $\Delta(a)=\Sigma a^{\prime} \otimes a^{\prime \prime}, \Delta(x)=$ $\Sigma x^{\prime} \otimes x^{\prime \prime}$, and $|-|$ and $\|-\|$ denote the "internal" and "external" degrees respectively. 
We can turn this left action into a right one by defining

$$
x \circ a=(-1)^{|x||a|}[-1]^{\circ\|x\|\|a\|} \circ a \circ x
$$

where $[-1]=\chi[1]$ with $\chi$ the conjugation. We will do this without further comment.

Denote by $\mathcal{C M}_{A_{*, *}}$ the category of $A_{*, *}$ coalgebraic modules with structure and bigrading preserving morphisms.

Next we need to recall the construction of the free $A_{*, *}$ coalgebraic modules generated by given supplemented coalgebras. Recall that a supplemented coalgebra $C_{*, *}$ is a cocommutative, coassociative coalgebra with counit over $R$ equipped with a map $\eta: R \rightarrow C_{*, *}$ with $\epsilon \eta$ the identity on $R$ (cf [10]). Denote by $\mathcal{S C}$ the category of supplemented coalgebras. Let $C_{*, *}$ be a supplemented coalgebra, then we have:

Proposition 2.4 [4] Denote by $\mathcal{D}$ the forgetful functor $\mathcal{C M}_{A_{*, *}} \rightarrow \mathcal{S C}$. Then it admits a left-adjoint which we will denote by $\mathcal{F}: \mathcal{S C} \rightarrow \mathcal{C M}_{A_{*, *}}$. Concretely $\mathcal{F}$ is given by $\mathcal{F}\left(C_{*, *}\right) \cong \operatorname{Sym}^{R}\left(A_{*, *} \otimes{ }^{R} C_{*, *}\right) / I$ where $I$ is the coalgebraic ideal generated by the elements of the form

$$
(a * b) \circ x-\sum(-1)^{\left|x^{\prime}\right||b|}\left(a \circ x^{\prime}\right) *\left(b \circ x^{\prime \prime}\right)
$$

and Sym is the symmetric power construction. Here we impose on $\mathcal{F}\left(C_{*, *}\right)$ the canonical algebra structure on Sym, we make it a coalgebra by extending the coalgebra structure of $C_{*, *}$, and the $A_{*, *}$ action is given by the formula

$$
a \circ\left(x_{1} \otimes \cdots x_{n}\right)=\Sigma\left(a^{\prime} \otimes x_{1}\right) \otimes \cdots \otimes\left(a^{(n)} \otimes x_{n}\right)
$$

where $\Sigma a^{\prime} \otimes \cdots a^{(n)}$ is the iterated coproduct on $a$.

With these preparations, we can state the definition of the coalgebraic tensor product.

Definition 2.5 [4] Let $M_{*, *}$ and $N_{*, *}$ be $A_{*, *}$ coalgebraic modules. Then we define $M_{*, *} \bar{\otimes}^{A, *} N_{*, *}$ to be the quotient of $\mathcal{F} \mathcal{D}\left(M_{*, *} \otimes{ }^{R} N_{*, *}\right)$ by $J$ where $J$ is the coalgebraic ideal generated by the elements of the form

$$
\begin{gathered}
u \otimes(x * y)-\sum(-1)^{\left|u^{\prime \prime}\right||x|}\left(u^{\prime} \otimes x\right) \mp\left(u^{\prime \prime} \otimes y\right), \\
(u * v) \otimes x-\sum(-1)^{|v|\left|x^{\prime}\right|}\left(u \otimes x^{\prime}\right) \bar{*}\left(v \otimes x^{\prime \prime}\right), \\
(a \circ u) \otimes x-a \circ(u \otimes x), \\
u \otimes(x \circ a)-(u \otimes x) \bar{\circ} a,
\end{gathered}
$$

where $\bar{o}$ and $\bar{*}$ are the structure maps of $\mathcal{F} \mathcal{D}\left(M_{*, *} \otimes^{R} N_{*, *}\right)$. 
To conclude the section we quote a couple more results that are only needed in the next section.

\section{Theorem 2.6 [4, Proposition 6.1] The bifunctor $\bar{\otimes}$ is right-exact.}

Proposition 2.7 [4] Denote by $\mathcal{M}_{S}$ the category of $S$-modules, and $A_{*, *}=R[S]$. The functor $R[-]: \mathcal{M}_{S} \rightarrow \mathcal{C M}_{A_{*, *}}$ is exact and commutes with the coproduct.

Note that the coproduct in $\mathcal{C M}_{A_{*, *}}$ is just the ordinary tensor product over $R$.

\section{Coalgebraic tensor product with a module-ring}

In this section we state and prove Theorem 1.1 when $A_{*, *}=R[S], N_{*, *}=R[T]$ where $S$ is a ring and $T$ an $S$-module. Throughout the paper, let $\Theta$ be a cocommutative and coassociative $R$-Hopf algebra (but not necessarily commutative). We say that $M_{*, *} \in \operatorname{Obj}\left(\mathcal{C M}_{A_{*, *}}\right)$ is a right (left, respectively) $\Theta-A_{*, *}$-coalgebraic module if $\Theta$ acts on $M_{*, *}$ on the right (left, resp.) so that both $\Delta_{M_{*, *}}$ and $\mu_{M_{*, *}}$ commute with $\Theta$-action, where $\Theta$ acts on $M_{*, *} \otimes M_{*, *}$ via the diagonal $\Delta_{\Theta}$, and the circle product with elements of $A_{*, *}$ commutes with $\Theta$-action. For the sake of concreteness, we will only consider the case of right actions unless otherwise specified. Now we can state:

Theorem 3.1 Let $A_{*, *}$ and $N_{*, *}$ be as above, and $M_{*, *}$ be a $\Theta-A_{*, *}$-coalgebraic module. Then $M_{*, *} \bar{\otimes}^{A, *} N_{*, *}$ becomes naturally a $\Theta-A_{*, *}-$ coalgebraic module.

Proof Let $T_{1} \rightarrow T_{0} \rightarrow T$ be a $S$-free presentation of $T$. Then the sequence

$$
M_{*, *} \bar{\otimes}^{A_{*, *}} R\left[T_{1}\right] \rightarrow M_{*, *} \bar{\otimes}^{A_{*, *}} R\left[T_{0}\right] \rightarrow M_{*, *} \bar{\otimes}^{A_{*, *}} N_{*, *}
$$

is exact in $\mathcal{C M}_{A_{*, *}}$ by Proposition 2.7. However, again by Proposition 2.7 the first two members of this exact sequence is just iterated tensor products of $M_{*, *}$ with itself, so they have a natural $\Theta-A_{*, *}$-coalgebraic module structure, and the map between them is easily seen to be compatible with the $\Theta$ action. The desired result now follows.

\section{Coalgebraic tensor product over a ring-ring}

In this section we state and prove Theorem 1.1 when $A_{*, *}=R[S]$, and $N_{*, *}$ is arbitrary. Namely we will show:

Theorem 4.1 Let $S$ be a ring, $M_{*, *}$ and $N_{*, *} \Theta-A_{*, *}$-coalgebraic modules, where $A_{*, *}=R[S]$. Then $M_{*, *} \bar{\otimes}^{A_{*, *}} N_{*, *}$ admits a unique $\Theta-A_{*, *}-$ coalgebraic module structure compatible with that on $M_{*, *} \otimes{ }^{R} N_{*, *}$. 
To prove this, we follow step by step the construction of the coalgebraic tensor product. Thus we will start from the free coalgebraic module generated by a coalgebra. So we need:

Definition 4.2 An $R$-supplemented coalgebra $C$ is called a $\Theta$-coalgebra if $\Theta$ acts on $C$ and the diagonal map $\Delta_{C}: C \rightarrow C \otimes C$ is compatible with $\Theta$-action where $\Theta$ acts on $C \otimes C$ via the diagonal $\Delta_{\Theta}: \Theta \rightarrow \Theta \otimes \Theta$.

Now we can state our first step, namely:

Lemma 4.3 Let $C$ be a $\Theta$-coalgebra and $S$ a ring. Then $\mathcal{F}(C)$ admits a unique $\Theta-A_{*, *}$-coalgebraic module structure compatible with that on $C$ where $A_{*, *}=R[S]$. Furthermore natural maps $C^{\otimes n} \rightarrow \mathcal{F}(C)$ are compatible with $\Theta$-action.

Proof The statement of the Lemma indicates how to define the $\Theta$-action on $\mathcal{F}(C)$. The uniqueness is also clear. We will proceed to show that this action is indeed welldefined. First of all there is no trouble in extending the $\Theta$-action on $C$ to $R[S] \otimes{ }^{R} C$. By the coassociativity and cocommutativity of $\Theta$, its action on $\operatorname{Sym}\left(R[S] \otimes{ }^{R} C\right)$ is well-defined. So it remains to show that the coalgebraic ideal $I$ in Proposition 2.4 is invariant under $\Theta$-action. However the defining relations of $I$ can be considered as the lack of the commutativity of the following diagram:

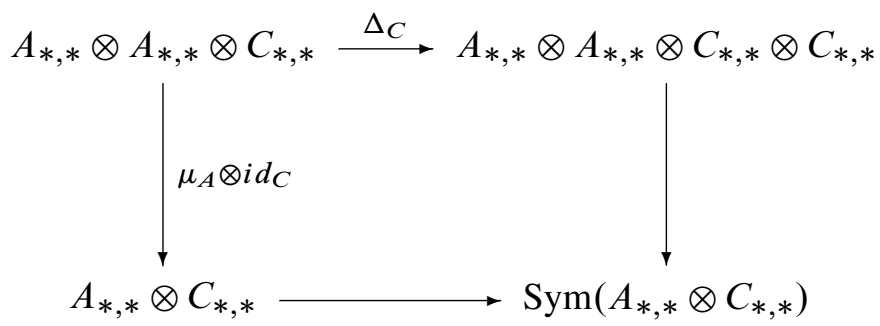

That is, $I$ is generated by the difference of the images by the two maps of elements at the top-left corner. However, our hypotheses imply that both top horizontal arrow and the left vertical arrow are compatible with $\Theta$-action, and we defined the action of $\Theta$ on $\operatorname{Sym}\left(A_{*, *} \otimes C_{*, *}\right)$ in such a way that the two other arrows become compatible with the $\Theta$-action. Thus we see that $I$ is invariant. Thus we can define an action of $\Theta$ on $\mathcal{F}(C)$. By construction it is compatible with the product. The compatibility with the coproduct follows from the compatibility of the diagonal of $C$ with the $\Theta$ action. The compatibility with the $A_{*, *}$-action is straightforward.

Proof of Theorem 4.1 Now we are ready to prove Theorem 4.1. Thanks to the Lemma we see that we can make $\Theta$ act on $\mathcal{F} \mathcal{D}\left(M_{*, *} \otimes^{R} N_{*, *}\right)$. Thus we only need to show 
that the ideal $J$ in Definition 2.5 is invariant under the $\Theta$-action. We will deal with the first type and the third type of elements, as the others are similar. The first type of elements correspond to the lack of commutativity of the following diagram:

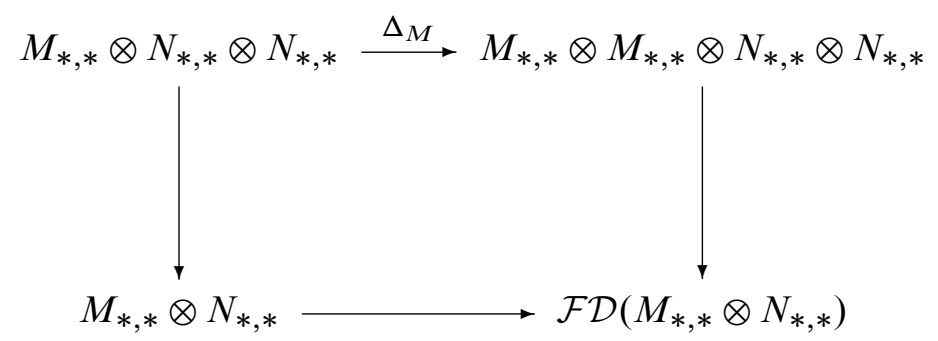

However, as in the proof of the Lemma, every arrow is compatible with $\Theta$-action. As to the third set of generators, they come from the lack of commutativity of the following diagram:

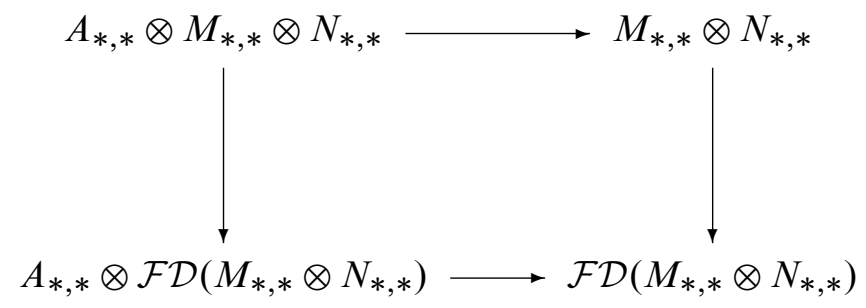

Here, the top horizontal arrow is compatible with $\Theta$-action by the hypothesis, other arrows commute with $\Theta$-action by the Lemma. Thus we have a well-defined action of $\Theta$ on $M_{*, *} \bar{\otimes}^{A_{*, *}} N_{*, *}$. It is easy to show that this action satisfies the required properties.

\section{The general case}

In this section we state and prove Theorem 1.1 in the most general setting. First we need the following.

Definition 5.1 Let $A_{*, *}$ be a coalgebraic ring over $R$ and $\Theta$ a Hopf algebra over $R$. We say that $A_{*, *}$ is a $\Theta$-coalgebraic ring over $R$ if all structure maps commute with the action of $\Theta$, where $\Theta$ acts on $A_{*, *} \otimes A_{*, *}$ via the diagonal $\Delta_{\Theta}: \Theta \rightarrow \Theta \otimes \Theta$. An $A_{*, *}$-coalgebraic module is called a $\Theta-A_{*, *}$-coalgebraic module if all structure maps (the star product, the coproduct, and the action of $A_{*, *}$ ) commute with the $\Theta$ action. 
Now we are ready to state:

Theorem 5.2 If $A_{*, *}$ is a $\Theta$-coalgebraic ring over $R$ and $M_{*, *}$ and $N_{*, *}$ are $\Theta-$ $A_{*, *}$-coalgebraic modules, then there is unique $\Theta-A_{*, *}$-coalgebraic module structure on $M_{*, *} \bar{\otimes}^{A, *} N_{*, *}$ that is compatible with the $\Theta$-action on $M_{*, *} \otimes{ }^{R} N_{*, *}$.

Proof This statement can be proved exactly in the same way as in the previous section, and is left as exercise to the reader.

Remark 5.3 When $A_{*, *}=R[S]$, the definition in this section coincides with that of the previous section if we let $\Theta$ act on $A_{*, *}$ through the augmentation map $\Theta \rightarrow R$.

Remark 5.4 (1) Let $\mathbf{E}_{*}$ be a graded ring space, and $\mathbf{F}_{*}$ an $\mathbf{E}_{*}-$ module. Denote $\mathcal{A}=H Z / p^{*}(H Z / p)$, the $\bmod p$ Steenrod algebra. Then $H_{*}\left(\mathbf{E}_{*} ; Z / p\right)$ is a $\mathcal{A}-$ coalgebraic ring and $H_{*}\left(\mathbf{F}_{*} ; Z / p\right)$ is a $\mathcal{A}-H_{*}\left(\mathbf{E}_{*} ; Z / p\right)$-coalgebraic module. Note that if $\mathbf{E}_{*}$ is a discrete ring (so that $\pi_{0}\left(\mathbf{E}_{*}\right)=\mathbf{E}_{*}$ ), then $H_{*}\left(\mathbf{E}_{*} ; Z / p\right) \cong Z / p\left[\mathbf{E}_{*}\right]$, thus we are in the situation of the previous section.

(2) The action of the Dyer-Lashof algebra $\mathcal{R}$ deserves some discussion. Let $E$ be a ring spectrum, $F, G E$-module spectra, $\mathbf{E}_{*} \mathbf{F}_{*}$ and $\mathbf{G}_{*}$ their associated infinite loop spaces. Then one easily sees that $H_{*}\left(\mathbf{F}_{*} ; Z / p\right)$ is a $\mathcal{R}-Z / p\left[\pi_{0}\left(\mathbf{E}_{*}\right)\right]$-coalgebraic module ( $\mathcal{R}$ acting on the left). However, one can't expect in general $H_{*}\left(\mathbf{F}_{*} ; Z / p\right)$ to be a $\mathcal{R}-H_{*}\left(\mathbf{E}_{*} ; Z / p\right)$-coalgebraic module since at the space level, the multiplication $\mathbf{E}_{*} \times \mathbf{F}_{*} \rightarrow \mathbf{F}_{*}$ is represented by maps which are not maps of infinite loop spaces (not even maps of loop spaces as a matter of fact). Nevertheless, it is still likely that $H_{*}\left(\mathbf{F}_{*} ; Z / p\right) \bar{\otimes} H_{*}\left(\mathbf{E}_{*} ; Z / p\right) H_{*}\left(\mathbf{G}_{*} ; Z / p\right)$ is equipped with a natural action of Dyer-Lashof algebra, as it is clearly a $\mathcal{A}-H_{*}\left(Q S^{0}\right)$-coalgebraic module, and in view of May's formula (see Cohen, Lada and May [2, page 15]) which relates the $H_{*}\left(Q S^{0}\right)$-coalgebraic module structure and the actions of $\mathcal{A}$ and $\mathcal{R}$ it is likely that under a suitable instability hypothesis, one can construct a $\mathcal{R}$ action from the $\mathcal{A}$ $H_{*}\left(Q S^{0}\right)$-coalgebraic module structure. We hope to come back to this subject in a future work.

\section{References}

[1] J F Adams, Lectures on generalised cohomology, from: "Category Theory, Homology Theory and their Applications, III (Battelle Institute Conference, Seattle, Wash., 1968, Vol. Three)", Springer, Berlin (1969) 1-138 MR0251716

[2] F R Cohen, T J Lada, J P May, The homology of iterated loop spaces, Lecture Notes in Math. 533, Springer, Berlin (1976) MR0436146 
[3] E Dyer, R K Lashof, Homology of iterated loop spaces, Amer. J. Math. 84 (1962) 35-88 MR0141112

[4] J R Hunton, P R Turner, Coalgebraic algebra, J. Pure Appl. Algebra 129 (1998) 297-313 MR1631257

[5] J R Hunton, P R Turner, The homology of spaces representing exact pairs of homotopy functors, Topology 38 (1999) 621-634 MR1670416

[6] T Kashiwabara, Homological algebra for coalgebraic modules and mod $p K$-theory of infinite loop spaces, K-Theory 21 (2000) 387-417 MR1828183 Special issues dedicated to Daniel Quillen on the occasion of his sixtieth birthday, Part V

[7] T Kashiwabara, W S Wilson, The Morava K-theory and Brown-Peterson cohomology of spaces related to BP, J. Math. Kyoto Univ. 41 (2001) 43-95 MR1844862

[8] J P May, Problems in infinite loop space theory, from: "Conference on homotopy theory (Evanston, Ill., 1974)", (D Davis, editor), Notas Mat. Simpos. 1, Soc. Mat. Mexicana, México (1975) 111-125 MR761724

[9] R J Milgram, The mod 2 spherical characteristic classes, Ann. of Math. (2) 92 (1970) 238-261 MR0263100

[10] D C Ravenel, W S Wilson, The Hopf ring for complex cobordism, J. Pure Appl. Algebra 9 (1977) 241-280 MR0448337

[11] NE Steenrod, Cohomology operations, and obstructions to extending continuous functions, Advances in Math. 8 (1972) 371-416 MR0298655

Institut Fourier, Université de Grenoble I

100 rue des maths, BP 74, 38402 St Martin d'Hères, France

Takuji.Kashiwabara@ujf-grenoble.fr

Received: 16 September 2009 Revised: 10 June 2010 